Questions

vives

\section{Questions Vives}

Recherches en éducation

$N^{\circ} 23 \mid 2015$

Évaluation et changement

\title{
Formation initiale des enseignants de langues : évaluation formative à visée certificative et/ou évaluation formative socialement partagée?
}

\section{Frédérique Longuet}

\section{(2) OpenEdition}

\section{Journals}

Édition électronique

URL : http://journals.openedition.org/questionsvives/1740

DOI : $10.4000 / q u e s t i o n s v i v e s .1740$

ISSN : $1775-433 X$

Éditeur

Université Aix-Marseille (AMU)

Édition imprimée

ISBN : 978-2-912643-47-6

ISSN : 1635-4079

Référence électronique

Frédérique Longuet, «Formation initiale des enseignants de langues : évaluation formative à visée certificative et/ou évaluation formative socialement partagée? ", Questions Vives [En ligne], Nº 23 | 2015, mis en ligne le, consulté le 20 avril 2019. URL : http://journals.openedition.org/ questionsvives/1740; DOI : 10.4000/questionsvives.1740

Ce document a été généré automatiquement le 20 avril 2019.

\section{(†)

Questions Vives est mis à disposition selon les termes de la licence Creative Commons Attribution Pas d'Utilisation Commerciale - Pas de Modification 4.0 International. 


\title{
Formation initiale des enseignants de langues : évaluation formative à visée certificative et/ou évaluation formative socialement partagée?
}

\author{
Frédérique Longuet
}

\section{Introduction}

1 Cet article questionne la formation initiale des enseignants de langues, tout en ayant une portée générale. La manière d'évaluer les compétences professionnelles en cours et/ou à la fin de la formation est un bon révélateur de la finalité institutionnelle visée. Le contexte français connait un changement profond de sa formation professionnelle avec le passage de l'Iufm (institut universitaire de formation des maitres) à l'Espe (école supérieure professionnelle de l'éducation). Nous proposons de questionner cette évolution en partant de l'analyse de l'évaluation formative à visée certificative, qui s'est concrétisée dans les années 2010, pour étudier, dans une deuxième phase et de manière prospective, une approche expérimentale de l'évaluation que nous nommons « évaluation formative socialement partagée ». Dans un premier temps, nous présenterons la problématique qui sous-tend notre argumentation. À partir de ce construit théorique, nous verrons si la démarche formative à visée certificative a permis de consolider une évaluation au service des apprentissages professionnels ou si la visée certificative a pris le dessus au détriment des apprentissages. Dans un deuxième temps, nous présenterons la deuxième approche que nous avons mise en place dans le cadre du master Espe Paris Sorbonne. Il s'agira de montrer concrètement ce qui constitue l'évaluation formative socialement partagée. La méthodologie d'analyse des observables s'inspire de l'analyse de discours, dont le but est de rendre compte des implicites énonciatifs à travers les marqueurs d'énonciation et les marqueurs dialogiques. Nous étudierons dans le premier cas quelques extraits de journaux de bord et dans le deuxième cas quelques exemples de 
dialogues au sein de blogues communautaires considérés comme des journaux collaboratifs.

\section{Contexte : entre nécessité certificative et nécessité formative}

2 Comme pour toute formation professionnelle initiale, l'enjeu est double dans la mesure où il s'agit de faciliter la construction de compétences professionnelles, mais aussi, et souvent surtout, certifier l'acquisition des compétences fondamentales définies par un référentiel professionnel. L'évaluation certificative externe a pris une place majeure, elle interroge la possibilité d'une réelle démarche formative en formation initiale (DuruBellat, 2009; Figari, 2006). Avec le développement de l'évaluation certificative par standards et par référentiel de compétences, comme c'est le cas pour l'enseignement des langues (Huver \& Springer, 2011), les frontières autrefois étanches entre le formatif et le sommatif sont devenues poreuses. Les tensions sont ainsi poussées à l'extrême entre une indispensable évaluation formative au service des apprentissages et une nouvelle injonction institutionnelle à harmoniser et contrôler le rendement du système de formation par le biais d'une évaluation certificative externe et standardisée. Ces difficultés et tensions ne peuvent que se lire dans les écrits professionnels proposés aux enseignants stagiaires. L'analyse des productions réflexives sur la construction et la production des savoirs et compétences professionnels des enseignants en formation initiale occupe logiquement une place de plus en plus significative (Allal, 2009 et 2013).

En France, le passage de la formation professionnelle en Iufm vers une formation de type master à l'université et dans les Espe offre une occasion intéressante de questionner les aspects formatifs de l'évaluation professionnelle à travers deux cas différents. Le premier cas est celui de l'Iufm Paris Sorbonne, département d'allemand (2009/2010). L'Iufm a mis en place une démarche mixte, démarche du praticien réflexif et visée certificative selon le référentiel de compétences. La démarche du praticien réflexif (Schön, 1993) a pour but de focaliser sur les apprentissages professionnels, c'est-à-dire la construction de compétences professionnelles dans et sur l'action. Par ailleurs, les Iufm se sont également inscrits dans le mouvement de clarification du métier d'enseignant en se dotant, à partir de l'arrêté de décembre 2006, d'un cahier des charges et d'un référentiel des dix compétences professionnelles. Dès lors, l'évaluation institutionnelle certificative a été intégrée à la démarche initiale formative et réflexive. Les futurs enseignants devaient documenter les compétences professionnelles définies par le référentiel, c'est-à-dire donner des preuves de conformité au modèle prescrit, à des façons de faire et d'être, qui constituaient les bonnes pratiques attendues (Longuet \& Springer, 2012). La trame proposée par l'Iufm pour les écrits servant à l'évaluation et à la validation internes était la suivante les stagiaires étaient encouragés à rédiger après chaque séance ou unité de cours un bilan à partir des 10 compétences avec, si possible, des commentaires réflexifs. Ces bilans étaient personnels et n'étaient pas remis au formateur. En accompagnement, le formateur, en général, proposait de voir comment documenter, avec des cas concrets, les 10 compétences dans la mesure où le «dossier de présentation " constituait l'écrit professionnel principal pour la validation (sélection de "preuves" déposées dans le dossier de travail pour faire apparaitre l'acquisition des 10 compétences visées). C'est par conséquent la conformité au référentiel qui l'emportait sur une réelle démarche réflexive professionnelle. L'évaluation finale était sommative, avec une grille des 10 compétences 
validées en acquis ou non acquis. La formatrice chercheure, pour sa recherche, a souhaité adopter une approche portfolio d'apprentissage. Les 7 stagiaires qu'elle accompagnait devaient ainsi tenir leur journal de bord. Le portfolio, ou journal de bord, à condition qu'il soit bien la propriété de l'enseignant stagiaire, peut constituer un outil au service des apprentissages et offre la possibilité de développer « un point de vue singulier » sur l'agir professionnel (Bucheton \& Chabanne, 2002, p. 11). Il s'agissait de ne pas s'enfermer dans un portfolio de présentation ou, pour reprendre Paquay (2004, p. 13), un «dossier de promotion » dont l'objectif est de vérifier si tout est bien acquis. Nos 7 stagiaires ont dû expliquer à leur tuteur qu'ils/elles allaient tenir un journal de bord personnel. Curieusement, quatre journaux ont été rédigés par les conseillers pédagogiques pendant que la stagiaire faisait cours. Deux journaux sont écrits par les stagiaires, mais un seul a été rédigé tout au long de l'année. Les stagiaires ont accepté de remettre à la fin de l'année (ils n'avaient pas été prévenus) leurs journaux de bord pour cette recherche. Nous analyserons quelques exemples de journaux de bord pour voir si l'instance énonciative, qui devrait être celle de l'enseignant stagiaire, se manifeste et exprime une vision du métier et du développement professionnel. Nous verrons ainsi si la visée certificative apporte des éléments positifs, comme l'affirment certaines recherches (par exemple Tessaro, (2015)), ou bien si, au contraire, on constate des effets négatifs dus à la visée certificative institutionnelle. Pour cela, nous utiliserons une analyse de discours afin de rendre compte des jeux énonciatifs (l'appareil formel de l'énonciation, pour reprendre Benveniste (1974)) qui expriment les jeux de discours (et de pouvoir) entre les experts, qui accompagnent ou savent et jugent, et les enseignants stagiaires qui tentent de se construire professionnellement en mettant au jour un style d'enseignant et une pensée didactique ou qui s'adaptent aux contraintes et prescriptions institutionnelles pour réussir la certification.

Le passage à une formation initiale universitaire en Espe (la mastérisation des formations) modifie inévitablement cette procédure institutionnelle en apportant d'autres tensions, par exemple entre les jeux de pouvoir de l'inspection et ceux des universitaires, mais aussi en donnant la possibilité de tester une autre démarche. Peut-on dans ce nouveau paysage envisager, et dans quelles conditions, une évaluation formative ouverte et bienveillante, favorable à l'émergence et au développement de nouvelles compétences professionnelle et universitaire? La mise en place des Espe et de la mastérisation de la formation initiale des enseignants devrait permettre de questionner les principes de l'évaluation formative. En tant que formatrice-chercheure, nous avons eu la possibilité, en 2010/2011, de concevoir un environnement formatif fondé sur une démarche collaborative, c'est-à-dire une approche de projet intégrant les Tice. Ce deuxième cas échappe en quelque sorte aux contraintes institutionnelles, même si le référentiel des 10 compétences demeurait le repère institutionnel indispensable. L'idée était de permettre aux enseignants stagiaires d'être des designers créatifs (Schön, 1992; Bucciarelli, 1994) constitués en communauté professionnelle (Wenger, 2005). Nous nous situons ainsi dans une approche sociale permettant l'émergence de compétences professionnelles négociées dans un cadre plus «informel " (Morrissette et Compaoré, 2012). Contrairement à la démarche cognitive individualisante du premier cas, nous nous inscrivons dans une perspective "considérant que le savoir-faire d'un praticien est en grande partie celui du collectif dont il est membre» (p.28). Il s'agit alors de repérer les traces d'une microculture professionnelle en construction. On conçoit dans ce cas l'évaluation formative comme une pratique sociale située. Huver \& Springer (2011) parlent d'évaluation alternative à l'évaluation dominante standardisée. Nous avons défini 
cette évaluation comme une évaluation socialement partagée (Longuet, 2012) qui s'inscrit dans un processus de socialisation professionnelle. Pour ces raisons, nous avons fait le choix de nous intéresser aux verbalisations professionnelles collectives, c'est-à-dire au dialogisme qui s'exprime grâce aux blogues communautaires que nous avons proposés. L'analyse de discours doit, dans ce cas, explorer le dialogisme des productions professionnelles de la communauté de pratique par le biais des marqueurs dialogiques. Les observables sont issus de 7 blogues communautaires, considérés comme journaux collaboratifs, qui regroupaient 21 stagiaires (nous disposons par ailleurs de 17 wikis et 9 forums). Notre posture de chercheure est essentiellement compréhensive. Il ne s'agit pas de proposer une méthode idéale qui ressemblerait à ce deuxième cas, mais de montrer, grâce à une étude énonciative et dialogique fine des verbalisations professionnelles, les traces de la construction identitaire professionnelle à l'œuvre dans une communauté de pratique. Nous pensons que cette construction s'appuie sur une évaluation formative qui se révèle en dialogues et en actions avec les autres et qui est socialement partagée par la communauté.

5 Nous présenterons successivement ces deux approches de l'évaluation formative en ayant conscience du risque de contraste et de dichotomie produit par la présentation. Les exemples choisis sont caractéristiques de l'ensemble des données recueillies et ne sont pas fabriqués pour les besoins d'une cause (pour plus de détails, voir Longuet, 2012). Cette recherche est de type exploratoire, inductive et qualitative; elle n'a pas pour visée de fournir un modèle à suivre mais de questionner l'impact des outils formatifs utilisés sur le développement professionnel.

\section{L'évaluation formative à visée certificative}

\subsection{Terrain et méthodologie}

$6 \quad$ Il s'agit de 7 journaux de bord rédigés par un groupe de stagiaires que nous avions sous notre responsabilité dans le module accompagnement. Nous avons décidé, pour les besoins de notre recherche, de nous inscrire dans une approche formative réflexive. Ce choix de recherche s'éloignait des pratiques en place à l'Iufm, tournées vers la nécessité de documenter le référentiel de compétences professionnelles afin de se faire certifier et habiliter. Le journal de bord vise à révéler le développement des compétences professionnelles des enseignants. Il offre aux stagiaires l'opportunité de construire une succession d'images de soi, de réalisation de soi par une réflexion personnelle sur les moments pédagogiques vécus. Selon Deum \& Vanhulle (2006, p. 8), l'écriture est un moyen de "revoir inlassablement des conceptions et compréhensions du réel et de soi ». Ainsi, les moments reformulés et matérialisés dans les journaux doivent permettre aux diaristes de développer une parole pour se construire une identité professionnelle.

$7 \quad$ L'analyse discursive qui suit repose sur quelques exemples tirés de notre corpus constitué de 7 journaux tenus par des enseignants stagiaires PLC2 (Professeurs lycées et Collège $2^{\mathrm{e}}$ année) germanistes en formation à l'Iufm de Paris. Elle cherche à identifier et analyser les marqueurs de subjectivité dans le discours pour voir si et comment le locuteur «se pose comme sujet » (Daille et al., 2009, p. 105). Le jeu des marqueurs de l'énonciation apporte un éclairage intéressant sur ce qui se passe réellement dans le discours normalement libre des enseignants stagiaires. 
Différents chercheurs ont souligné « l'impersonnalisation » (Gomez, 2001, pp. 88-89) des enseignants stagiaires liée à un formatage pédagogique par les conseillers pédagogiques, formateurs, et inspecteurs. La formation proposée consiste dans ce cas à faire appliquer un modèle pédagogique défendu par l'institution. En choisissant une approche réflexive, nous avions pensé qu'il serait possible, pour les stagiaires, de développer une écriture impliquée, moyen pour se libérer des modèles imposés, afin de se construire une identité professionnelle propre. L'analyse énonciative du «sujet parlant » (Rabatel, 2008, p. 370) s'est ainsi imposée. Nous allons voir que l'on assiste, dans ces journaux, à « l'effacement énonciatif» (Rabatel, 2004, p. 4 ; Vion, 2001, p. 334) du «sujet parlant». L'analyse des indicateurs de subjectivité et de position énonciative dans les journaux de bord aboutit à une caractérisation du registre discursif des stagiaires et des conseillers pédagogiques.

\subsection{Un « simulacre énonciatif »}

Nous allons nous intéresser aux deux seuls journaux de bord réellement rédigés par les stagiaires. Les dates prouvent qu'il s'agit bien d'une écriture au fil du temps propre au journal, mais la posture énonciative choisie confère aux journaux une dimension inhabituelle. En effet, le «sujet parlant » ne s'implique pas dans l'acte d'énonciation. Il choisit de se retirer de l'acte énonciatif en rapportant les propos tenus par le conseiller pédagogique ou le formateur Iufm. On assiste ainsi à une désimplication du "sujet parlant " par l'objectivation de son discours. Les traces de subjectivité, les « embrayeurs " ou déictiques (Charaudeau et Maingueneau, 2002, p. 211), sont peu nombreuses. Elles renvoient au discours du conseiller pédagogique ou au discours du formateur Iufm. Le "sujet parlant» cherche ainsi à supprimer toute marque de "source énonciative identifiable » (Vion, 2001, p. 334). Ce « simulacre énonciatif» ou « jeu » joué par le « sujet parlant» (Charaudeau, 1992, p. 650) mérite d'être analysé pour comprendre le choix énonciatif des diaristes.

Dans ces deux journaux, l'énonciation repose essentiellement sur une série d'injonctions du conseiller pédagogique ou du formateur Iufm. Ces injonctions traduisent l'obligation de faire, d'exécuter les ordres. Le "sujet parlant» rapporte les ordres du conseiller pédagogique qui contraint le stagiaire à reproduire un modèle. L'infinitivisation des verbes dans le discours rapporté signale l'effacement du stagiaire. Elle permet au « sujet parlant» de mettre en scène le conseiller pédagogique ou le formateur Iufm sans intervenir. Le stagiaire, «sujet parlant », devient la deuxième personne de l'énonciation, un « tu » désigné par le conseiller pédagogique. Cicurel (1994) écrit à ce sujet, lors d'une analyse du discours didactique de l'enseignant : «l'injonction établit une relation entre les participants par le biais d'une action souvent verbale que doivent exécuter les apprenants ».

11 L'injonction se manifeste dans le journal de bord des stagiaires par l'usage de verbes au mode infinitif et le recours à l'exclamation: « exploiter », «manipuler!», « interroger ceux qui discutent», etc. Elle est parfois accompagnée d'un pictogramme signalant un danger. L'ordre est alors doublé d'une mise en garde. L'injonction est également renforcée par des interdictions véhiculées à travers l'usage d'adverbes de négation totale : " pas de flottement en début : prise en main », « ne pas interrompre », « ne jamais négocier », « pas de traduction », « pas sous pression ».

Enfin, les ordres et les interdictions sont intensifiés par le recours aux modalisateurs comme les dépréciatifs « trop », « plus » ou « moins » : « support donné trop tôt », « trop 
dans le dire ", «trop de mots ", «trop de vocabulaire ", " moins de questions ", " être plus méchante». Seules les injonctions énoncées par le formateur Iufm lors de la visite conseils sont accompagnées d'appréciations positives: «bonne prise en main », «bon déplacement », « positif reformulation », «bon relationnel ».

Quelques déictiques apparaissent dans les deux journaux mais ils ne correspondent pas au point de vue du «sujet parlant", le stagiaire. Deux énoncés dans un des journaux contiennent des indices de personnes à la première personne: «comment faire pour qu'ils m'écoutent, qu'ils soient contents de parler », «(pictogramme danger) ich bin kein Wörterbuch» («Je ne suis pas un dictionnaire»). Le pronom personnel «m'" renvoie au « je » de l'action pédagogique; les pronoms personnels « ils » aux élèves. L'interrogation formulée par le stagiaire suite aux deux ordres donnés par le conseiller pédagogique découle directement des deux injonctions du conseiller pédagogique: "les mettre au travail avec ce qui les intéresse », «trouver l'accroche ». Elle n'est donc pas le fruit d'une réflexion du stagiaire. Elle est formulée par le conseiller pédagogique et reformulée par le stagiaire pour l'intérioriser. Le pronom ich, quant à lui, renvoie au stagiaire qui n'est pas à l'origine de la réflexion. Le stagiaire cherche à assimiler la critique du conseiller pédagogique en la reformulant : «je ne suis pas un dictionnaire ». Quatre autres énoncés dans un journal renferment également la marque énonciative du stagiaire : «je dois les mettre en concertation", "je n'évalue pas assez souvent», "je n'ai pas vu ceux qui bavardent tout le temps », "Je leur ai dit de fermer les livres». Dans ce cas comme dans l'autre, les paroles sont rapportées par le stagiaire pour qu'il puisse se les approprier. Le point de vue énoncé par le « sujet parlant » n'est pas le sien.

Dernier exemple, la «désinscription énonciative» (Rabatel, 2004, p. 4) par le discours rapporté permet de mettre en scène un autre énonciateur, l'inspecteur. En effet, le conseiller et le formateur Iufm mentionnent le nom de l'inspecteur pour marquer le degré de véracité de leur énoncé. Le nom de l'inspecteur rappelle implicitement le titre et les fonctions des uns et des autres (Rouveyrol, 1999). Le degré de véracité du discours du conseiller pédagogique et du formateur est, par ailleurs, accentué par le recours à la typologie. Le nom de l'inspecteur est écrit en lettres capitales. Il est suivi du pictogramme danger et de ses exigences professionnelles : la discipline, le lexique, les contrôles. Son nom est également associé à la phonologie dans le premier journal : «phonologie !».

L'exemple ci-dessous illustre le simulacre énonciatif révélé par l'analyse énonciative des journaux de bord: 


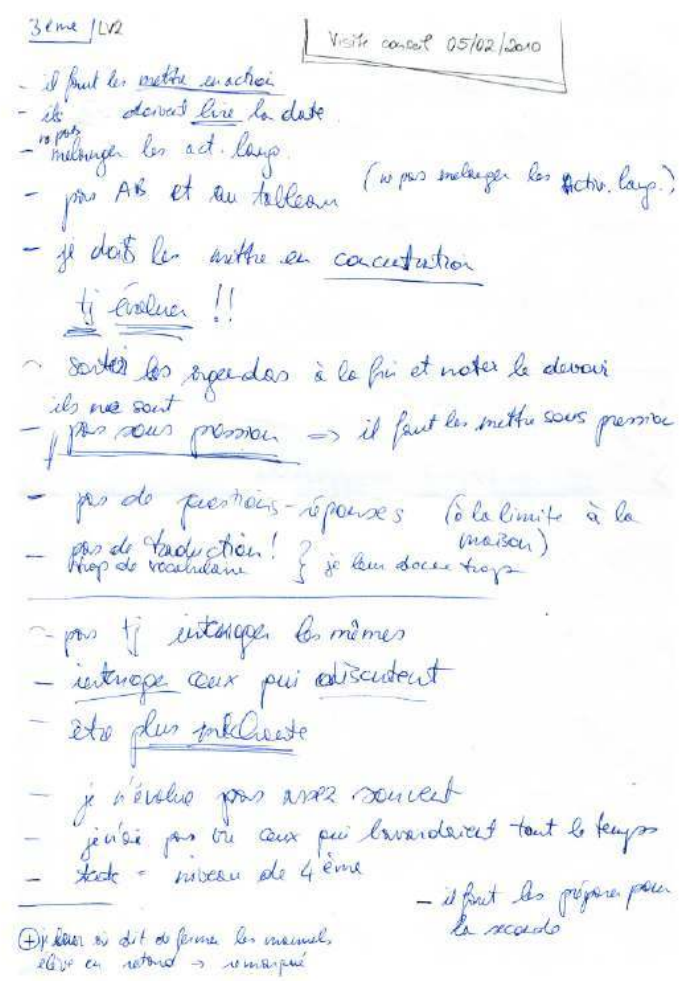

\subsection{Une prise de pouvoir énonciative et évaluative}

Nous nous intéressons pour cette analyse aux cinq journaux rédigés par les conseillers pédagogiques pendant que les stagiaires enseignent. Ils se différencient des deux précédents par leur situation d'énonciation. Ils sont datés et écrits à la première personne mais le « sujet parlant » n'est pas le stagiaire.

L'énonciation s'organise autour de la description de l'agir professionnel du stagiaire par le conseiller pédagogique et des remarques, interrogations ou jugements du conseiller pédagogique. Le conseiller pédagogique devient le « sujet parlant ». Il se met en scène et il met en scène un interlocuteur à qui il adresse des ordres, des recommandations, des critiques, des conseils. On est donc dans une communication fictive. Il y a un simulacre de communication avec une tierce personne absente de l'interlocution. À aucun moment le stagiaire n'est à l'origine d'une parole. Seul le conseiller pédagogique réfléchit sur l'agir professionnel observé. Sa présence est marquée par les indices personnels à la première personne, par les modalisateurs évaluatifs attachés aux énoncés à la deuxième ou troisième personne ou par l'emploi de tournures impersonnelles comme « il aurait fallu ».

Même si les indices personnels à la première personne sont beaucoup moins nombreux que les indices personnels à la deuxième ou troisième personne, le "sujet parlant » est omniprésent. Les verbes d'opinion associés aux actions décrites tels que "je pense que ", «je reste convaincue », «je crois » reflètent la réflexivité du conseiller pédagogique et non celle du stagiaire.

Les indices personnels sont quasiment systématiquement accompagnés du jugement évaluatif du "sujet parlant», ce qui lui confère un caractère dominant. Chaque geste 
professionnel est validé ou refusé. Les appréciations positives telles que "très bien ", «OK » ou dépréciatives telles que «non!» montrent que chaque geste professionnel du stagiaire est accepté ou refusé par le conseiller. L'intensité du jugement de valeur du conseiller pédagogique est marquée par les exclamatifs et les interjections : "OK", «non! », « cela ne fait rien!». Le pouvoir évaluatif du conseiller est également renforcé par la justification systématique de ses propos, ce qui n'appelle pas au dialogue: «je trouve dommage (...) car (...) ». En effet, le stagiaire, privé de la parole, ne peut pas interroger ses choix pédagogiques et développer son point de vue.

Il n'y a donc pas d'argumentation co-construite selon la définition de Grize (1990, p. 40) : « Telle que je l'entends, l'argumentation considère l'interlocuteur, non comme un objet à manipuler, mais comme un alter ego auquel il s'agira de faire partager sa vision. " Le stagiaire ne peut pas se construire une opinion sur le métier, il ne peut qu'adhérer au point de vue du conseiller pédagogique.

21 Enfin, les recours au conditionnel passé, aux injonctions ou à la tournure impersonnelle au présent «il faut» du «sujet parlant » dans l'ensemble des journaux soulignent la volonté du conseiller pédagogique de contraindre le stagiaire à enseigner tel qu'il le préconise, tel qu'il pense que l'institution le souhaite. Le conseiller présente l'action comme obligatoire ou non permise. En effet, les verbes pouvoir, devoir, falloir au conditionnel passé expriment les regrets et les reproches du conseiller pédagogique. Ils font écho aux injonctions des conseillers pédagogiques dans les précédents journaux.

L'extrait du journal de bord ci-dessous atteste de la prise de pouvoir énonciative du conseiller pédagogique :

Illustration 2 : Extrait corpus, journal de bord (Longuet, 2012, p. 335)

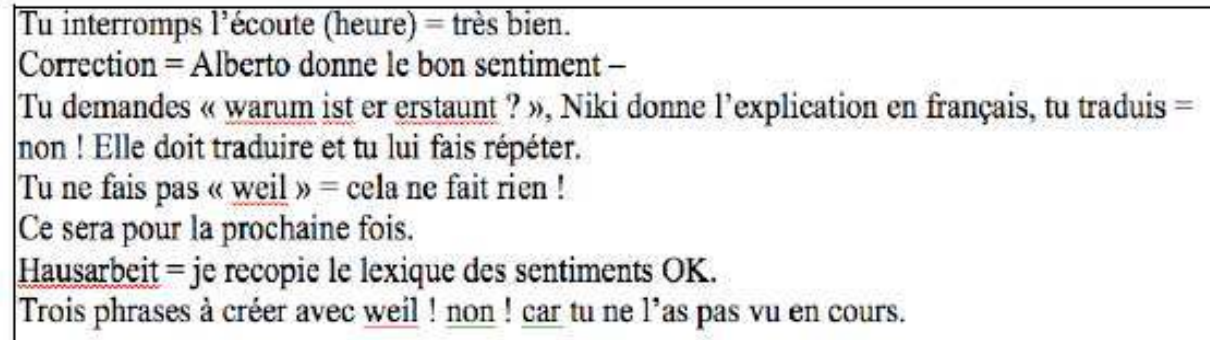

\subsection{Bilan intermédiaire : un anti-modèle d'évaluation formative et une parole confisquée}

23 D'autres exemples pourraient compléter ce tableau qui peut sembler caricatural. Bien entendu, cette analyse n'a aucune valeur généralisante. Elle ne concerne que ce terrain particulier, elle est donc située et spécifique. On ne peut en conclure que cette situation soit la règle générale. L'évaluation formative à visée certificative, dans ce cas, apparait comme un "anti-modèle» de la construction identitaire sociale et professionnelle. L'« effacement énonciatif ", choisi ou contraint, permet la mise en scène d'un discours polyphonique doxique. Le point de vue de Rabatel (2004, p. 10) sur la sous énonciation s'applique parfaitement : « la sous énonciation » du "sujet parlant » « exprime un PDV » (point de vue) «dominé en référence/révérence à une autorité par politesse, respect, admiration, soumission, libre adhésion, aliénation ou feintise, etc. ». Le stagiaire, devenu volontairement «sous énonciateur », dresse, par feinte ou soumission, un tableau cruel 
de ce type de formation institutionnelle. Il laisse entendre, voir, sans livrer explicitement son point de vue. d'évaluation, transmise par ce discours, ne peut pas permettre aux futurs enseignants de se construire positivement. On a du mal à trouver des traces d'une « modération sociale " qui devrait "améliorer la qualité des pratiques» professionnelles (Tessaro, 2015). L'artéfact journal de bord, en tant qu'outil de production d'une pensée professionnelle susceptible de servir de tremplin à une médiation sociale, entre l'expert et le novice, entre l'expert et le groupe, entre les membres du groupe, est, dans notre cas, détourné de son objectif. Il en va de même de la démarche du praticien réflexif. Aussi exagéré que cela puisse paraitre, il n'en est pas moins vrai que ces usages négatifs sont possibles si les accompagnateurs professionnels n'ont pas la formation nécessaire pour organiser une médiation et une modération sociales et compréhensives (Mottier Lopez et al., 2012). C'est bien la visée certificative qui domine les pratiques évaluatives au détriment d'une démarche d'évaluation formative.

\section{Une évaluation formative socialement partagée}

\subsection{Web 2.0, dialogisme et évaluation sociale}

Nous estimons que la construction identitaire professionnelle résulte de l'interaction sociale liée à une «intention collective» (Gilbert, 2008, p. 904 ; Perreau, 2010, p. 216) : créer ensemble. Cette optique nous invite à comprendre comment la construction identitaire professionnelle émerge des interactions sociales dans le cadre de la création 
de ressources didactiques pour l'apprentissage des langues. Les artéfacts du web 2.0 sont exploités dans la mesure où ils génèrent une dynamique sociale (Dillenbourg, Poirier \& Carles, 2003). Le forum, le wiki et le blogue communautaire constituent des médias sociaux interactifs impliquant un "co-engagement» (Gilbert, 2008, pp. 900-904) et une action sociale partagée qui permettent de dépasser la dimension individuelle de l'écriture propre aux journaux de bord.

À cet effet, l'environnement numérique d'apprentissage doit accorder une place centrale au sujet social dans la construction de l'identité professionnelle. Une culture partagée (Wenger, 2005) se crée au sein de la communauté de pratique. Chaque sujet social prend en main son développement personnel et professionnel avec et grâce à ses pairs. Le web 2.0, qui porte les communautés grâce aux artéfacts sociaux, nous place au cœur de l'intersubjectivité, du dialogisme et de l'évaluation sociale. Pour Voloshinov (2010), le langage est par essence social et intersubjectif. Il s'actualise dans l'interaction sociale. Bres (1998) souligne par ailleurs que "Bakhtine posait dans toute sa force l'autre en discours à travers le concept de dialogisme ». Bres poursuit en disant que "la réalité langagière de la langue en discours, c'est le dialogue sous sa double forme»: "dialogisation interdiscursive» et "dialogisation interlocutive». Cette double orientation vers ce qui a déjà été dit par d'autres et ce que l'on va dire permet de rendre compte du contexte intersubjectif. L'énonciation convoque des paroles plurielles et se construit sur "une compréhension-réponse». Cette manière de "comprendrerépondre » n'est pas simplement une façon de parler mais révèle une façon « d'écouterapprécier », donc de dialoguer.

Dans cette vision du dialogisme, il n'y a pas d'énonciation sans appréciation, sans évaluation. Elle s'opère dans la compréhension-responsive active des locuteurs. L'évaluation est de ce fait interne au processus dialogique qui met en scène des voix. Elle est, par conséquent éminemment sociale. Bres \& Mellet $(2009$, p. 6) disent que tout énoncé est habité par l'énonciateur et l'énonciataire mais également par d'autres voix, d'autres discours qui le «feuillettent énonciativement ». L'évaluation joue donc un rôle essentiel dans le processus de construction de sens de la langue (Boutet, 1994). On ne peut pas "construire d'énonciation sans orientation appréciative", "évaluer est une opération qui supporte toute énonciation »(Peytard, 1990, p.16). Toute évaluation devrait ainsi être « socialement partagée ».

\subsection{Terrain et méthodologie}

31 Pour ce deuxième cas, le corpus analysé est tiré d'une expérimentation en master mobilité avec 21 futurs professeurs des écoles. Il leur est demandé de concevoir en groupes, en négociant, un projet TICE et éventuellement de le mettre en œuvre lors du stage en mobilité. Ces groupes, grâce aux outils du web 2.0, se constituent en communautés de pratique autour de leur projet (e-book et ethnoblogue). On assiste dans ce cas à une perspective bien différente qui nous place dans un dialogisme réel. Ce deuxième cas va nous permettre d'explorer une autre piste d'évaluation formative pour la formation professionnelle. On se situe dans un cadre social avec une communauté de pairs qui construisent ensemble en action leur nouvelle identité et évalue socialement les compétences mises en œuvre dans le cadre du projet. Nous n'analyserons pas l'ensemble $\mathrm{du}$ corpus (blogues, wikis, forums) mais uniquement quelques extraits significatifs tirés 
des blogues communautaires considérés comme journal de bord de la communauté (pour une analyse détaillée voir Longuet (2012).

Que ce soit dans les forums ou les blogues, la communauté s'exprime à travers les déictiques on / nous qui encadrent le «je » dominant. La valeur du déictique «je » fait écho à la valeur inclusive du pronom «nous » issu de « l'entité nouvelle » (Gilbert, 2008, p. 903) créée par le co-engagement et du pronom «on» fortement utilisé. La quasi totalité des occurrences de « on » renvoie à la valeur inclusive du pronom « on », valeur qui inclut «le sujet de l'énonciation" "dans la composition interne de «on", avec d'autres » (Boutet, 1994).

L'analyse des échanges relevés sur les 9 blogues (185 billets et autant de commentaires) consiste à repérer les marqueurs d'opération dialogique, plus précisément les marqueurs du positionnement intersubjectif dans la dialogisation interdiscursive et interlocutive émanant des échanges favorisés par le web 2.0. Nous nous inspirons pour cela de l'approche méthodologique proposée par Bres afin de mesurer l'impact de l'évaluation sociale sur le développement des compétences professionnelles des enseignants de langues. Elle porte en particulier sur les liens dialogiques qui s'établissent entre les locuteurs, sur la variation du positionnement intersubjectif des futurs enseignants de langues.

\subsection{Une construction professionnelle à travers un dialogisme interlocutif : une communauté professionnelle en émergence}

Les membres de la communauté forment « un ensemble unitaire » (Perreau, 2010) qui fabrique des connaissances que nous proposons d'analyser à travers un marqueur dialogique, l'interrogation. Les interrogations portent sur la mise en place de l'histoire ainsi que sur la mise en œuvre de la langue et de la culture dans le scénario créé. Elles concernent la conception des personnages et l'intrigue, l'espace-temps, la dimension culturelle et interculturelle, la langue et le rôle du visuel dans l'apprentissage des langues. Ces premières interrogations font apparaitre les composantes de base de l'interaction sociale nécessaires à l'apprentissage par tâches. Elles révèlent une didactique en acte en cours d'élaboration.

L'interrogation ne correspond donc pas à une simple demande d'information. Elle exprime "de l'incertitude » et elle "fait appel à une réponse ", ce qui lui confère une dimension dialogique (Therkelsen, 2009, p. 114). Elle a pour fonction de provoquer la réaction des membres de la collectivité, de solliciter un avis ou encore de mettre en débat des idées. Elle équivaut à une "demande de dire» (Quillard, 2001, pp. 59-60), à une ouverture aux différents points de vue que Lehti (2010, p.5) nomme «expansion dialogique ». Cette expansion dialogique à travers l'interrogation revêt différentes formes. Elle peut être déclenchée par un énoncé interrogatif ou déclaratif, exprimer une demande liée à un "non-savoir certain » ou à un "savoir incertain » (Therkelsen, 2009, pp.114-115). Les questions peuvent être adressées directement à la communauté de pratique dans sa globalité pour susciter des réactions. Elles représentent alors « la forme la plus manifeste du dialogisme interlocutif» (Lehti, 2010, p.6). L'extrait ci-dessous montre que la communauté de pratique naissante développe des compétences professionnelles à partir de la circulation d'idées dialoguées : 


\title{
Des nouvelles après un long silence :D
}

\author{
Alors après cette lo00000000000ngue période de trêve, voici quelques nouvelles de \\ notre e-book \\ Nous avons bien bossé à distance ce matin. Nous avons chacune fait et presque \\ terminé nos parties respectives sur lilie de Ré et Toulouse. \\ Nous avons mis le storyboard au présent. Nous avons aussi presque toutes nos \\ photos (ainsi que leur sourcellil)
}

Par contre, nous nous posons une question Nous avons fait le choix de ne pas

animer nos personnages, mais de les incruster à nos photos. Nous nous

demandons même à présent si nous voulons vraiment mettre des personnages.

Nous trouvons que ga gache les photos.

A votre avis?

Publié par Delphine à $11+46 \quad 3$ commentaires:

M $\mathrm{E} \in \mathbf{f}$ ( $\mathrm{G+1}$ Recommander ce contenu sur Google

Le processus de création didactique émerge au fil du temps. Il s'accommode de contraintes technologiques, pédagogiques et didactiques. En effet, les étudiants doivent créer un livre numérique interactif dans lequel l'apprenant intervient pour construire lui-même son apprentissage à partir de la démarche ethnographique ou à partir d'une animation 2D. L'interactivité pensée comme un moteur créatif, un véritable moyen d'innover rompt avec une pédagogie traditionnelle monodirectionnelle. De ce fait, les membres de la communauté de pratique, confrontés à des problèmes émergeant de la contrainte créatrice (prise en compte de l'enfant dans le cadre de la mise en place de l'histoire, langue comme outil de création pour l'apprenant, choix des outils TICE, prise en compte de la mobilité et donc de l'interculturel) s'intéressent à la création des autres pour faire progresser la leur. Chaque étape de la création ou chaque création en soi (dessin, vidéo) est soumise à l'appréciation de la communauté.

\subsection{Micro culture didactique partagée et médiation sociale}

Contrairement au modèle Iufm, les appréciatifs positifs sont dominants et vont au-delà d'une appréciation de façade que l'on trouve sur la toile. Ils sont renforcés par une analyse critique $d u$ "faire", une demande d'information complémentaire ou des suggestions. La capacité d'évaluation des membres de la communauté de pratique progresse et se consolide. Les commentaires montrent qu'ils sont capables d'analyse critique positive et de prise de recul. Ainsi, de nouveaux marqueurs dialogiques comme le détachement, le clivage, la concession argumentative apparaissent. Ils s'ajoutent aux marqueurs dialogiques précédemment rencontrés. Dans l'exemple qui suit, l'énonciateur du commentaire ouvre le débat sur les « exercices » proposés par un membre du groupe à intégrer dans la ressource numérique :

Illustration 4 : Extrait corpus blogue, http://fle-montessori.blogspot.fr/ (Longuet, 2012, pp. 231-232)

- [E2] Personnellement je trouve que les exercices sont un peu «formels» ce serait bien d'introduire les questions au fur et à mesure non? par exemple ds une situation au marché, 
mettre une petite consigne à la fin du style « donne quelques noms de fruits et légumes que tu vois sur l'image » ou qqe chose comme ça.

- [e1] Oui c'est vrai qu'on a pas encore vraiment parlé de comment on allait introduire ces exercices... questions à la fin d'une situation particulière, page réservée aux exercices (façon livres scolaires).. ? Bonne remarque $\mathrm{R}$, à voir avec ma team ${ }^{\wedge}$

- [E4] Bonsoir à toutes, j'ai regardé le site que tu nous as recommandé et je trouve que les items des exos sont vraiment intéressants mais difficilement aisés à replacer dans le contexte. Je crois que effectivement nous devrions créer un petit marché par exemple puis cliquer sur les légumes et écrire ce que c'est ou bien replacer les étiquettes avec le nom des légumes déjà inscrits...

- [E2] Si je peux me permettre, vous pouvez prendre une photo de stand de marché ou le dessiner et y placer par exemple des étiquettes carottes $5 €$ le kilo ou qch comme ça ? enfin qch de vraiment authentique pour véhiculer the real life française.

L'énonciateur [E2] se démarque du discours didactique qui sous-tend les choix de [e1] pour construire la compétence communicative langagière en qualifiant les " exercices » de « formels ». Elle rejette la notion d'exercice en formulant une tâche : « donne quelques noms de fruits et de légumes que tu vois sur l'image ». La proposition de l'énonciateur [E2] contenue dans l'énoncé déclaratif à valeur illocutoire interrogative « ce serait bien d'introduire les questions au fur et à mesure non? » suivie de l'exemple de la «situation de marché » oriente la réponse de l'énonciataire vers une réponse positive. Elle introduit une approche didactique de type actionnel qui s'oppose à l'approche didactique structuraliste traditionnelle de [e1]. Pour l'énonciateur [E2], les apprenants qui interviendront dans l'album interactif construiront la langue dans l'action en contexte social. Or les exercices trouvés sur le net sont des exercices structuraux décontextualisés qui ne favorisent pas la réflexion sur la langue à partir du contexte d'utilisation de la langue mais l'acquisition d'automatismes. Ils ne sont donc pas en adéquation avec le projet de littérature interactive. Cet implicite apparait clairement dans les propos de l'énonciateur [E4] qui juge les exercices intéressants «mais difficilement aisés à placer dans le contexte». L'opposition concessive marquée par «mais» laisse entendre que l'énonciateur [E4] s'oppose à l'idée que les exercices sont adaptables au projet uniquement parce qu'ils sont jugés intéressants. L'énonciateur [E4] approuve alors la proposition didactique de l'énonciateur [E2] en la reformulant : « créer un petit marché ». Les recherches soumises à l'appréciation des membres de la communauté de pratique conduisent à une prise de conscience didactique: une combinaison d'approches didactiques opposées est problématique.

Cet exemple de dialogue entre les membres de la communauté de pratique montre qu'ils acquièrent de l'expertise dans la confrontation aux autres, ils sont porteurs de discours professionnels pluriels. Les membres se positionnent comme des évaluateurs professionnels qui fondent leur évaluation à partir de la mise en relation des discours. L'expertise acquise permet aussi des critiques directes qui ne sont pas que consensuelles.

Le blogue, grâce au dialogisme, fournit ainsi un cadre favorable à la socialisation professionnelle. Chaque membre de la communauté est un pair, égal aux autres, capable de jugements. L'évaluation sociale partagée est inhérente aux échanges de la communauté. Elle fonde la construction identitaire professionnelle dans sa dimension sociale et individuelle. 


\subsection{Bilan intermédiaire : une évaluation formative informelle et socialement partagée}

41 La communauté de pratique décrite trop brièvement est le lieu d'une éclosion de paroles indispensables à la construction identitaire et à l'évaluation socialement partagée. Le discours général entremêle des voix personnelles qui révèlent un «savoir perceptuel » aux voix du discours didactique historique à travers le discours spécialisé ou "savoir transmis » (Carcassonne, 2010, pp. 8-11). Cet enchevêtrement des voix favorise ainsi l'émergence d'identités professionnelles socialement construites. L'évaluation formative et qualitative se situe au sein même du dialogisme et, par conséquent, au sein de toute communauté de pratique motivée par un but commun. L'évaluation qualitative est inhérente et naturelle à toute communauté sociale. Il est donc indispensable de permettre l'expansion dialogique nécessaire à l'expression de cette aptitude à apprécier l'action. L'éclosion de paroles rapidement présentée et l'incontournable présence de l'intersubjectivité dans le discours s'opposent à la confiscation de la parole que nous avons observée dans le cas précédent.

L'évaluation qualitative des compétences professionnelles en construction ne peut se faire que de manière dialogique au sein d'une communauté de pratique professionnelle apprenante dans le cadre d'un processus de référentialisation (Figari, 1994). Elle peut difficilement se faire de manière externe dans une relation d'expert à novices et à partir de standards prédéfinis par un référentiel de compétences institutionnel. Nous ne partageons donc pas les points de vue sur les possibilités de réduire les tensions (Mottier Lopez \& Crahay, 2009) entre approche formative des apprentissages et approche institutionnelle certificative. Nous pensons que la démarche d'évaluation formative socialement partagée est suffisamment documentée pour constituer des preuves d'un développement professionnel conforme à un référentiel de compétences non contraignant.

\section{Discussion et perspectives}

43 Cette étude a permis de confronter deux approches de l'évaluation formative professionnelle. Le tableau peut sembler trop dichotomique mais l'analyse de discours de type énonciation a permis de bien faire ressortir les traces contrastées de deux portraits professionnels. L'évaluation institutionnelle, par référentiel et standards, a le désavantage, si les évaluateurs n'ont pas de formation et s'ils s'imaginent comme des garants des bonnes pratiques professionnelles, d'offrir aux enseignants une liste de mauvaises conduites à éviter. Nous avons pu voir que dans ce cas, les enseignants stagiaires n'ont pas droit à la parole, tout développement professionnel est alors nié. Nous risquons même de conforter des pratiques évaluatives négatives que les enseignants stagiaires ont rencontrées lorsqu'ils étaient élèves. Dans le deuxième cas, l'évaluation formative socialement partagée, qui donne aux enseignants un certain pouvoir et qui les traite en réels acteurs en développement, s'inscrit dans une vision alternative à ce modèle institutionnel dominant. Il s'agit de favoriser une prise de conscience et une prise de confiance dans le développement de l'expertise didactique. Ce type d'évaluation se dévoile à travers la constitution d'une communauté de praticiens qui s'écoute et s'entraide. Elle s'inscrit dans la démarche de "productions de savoirs partagés " 
(Morissette et al., 2012). Elle exploite également le potentiel collaboratif de l'ère numérique avec les réseaux sociaux que les jeunes pratiquent. L'espoir porté par cette expérience va dans le sens d'un possible progrès que la mastérisation pourrait porter.

Cependant, le changement de modèle esquissé dépend d'un autre projet social d'éducation qui vise à former des enseignants ouverts au dialogue et à l'innovation, mais aussi des jeunes citoyens acteurs sociaux en développement, sensibles à la créativité dans sa dimension sociale. Il faudrait pour cela que la chaîne de décision institutionnelle, du conseiller à l'inspection, puisse être modifiée. Il faudrait que l'on ne se contente plus d'enfermer l'enseignant dans son statut administratif d'agent de l'État soumis aux prescriptions règlementaires. L'enseignant devrait être considéré comme un professionnel doué d'une attitude réflexive qui éclaire les savoirs et savoir-faire collectifs, capable d'évoluer dans une société tournée vers le numérique et l'intelligence collective.

Malheureusement, l'évaluation et la certification professionnelles du nouveau master MEEF (master métiers de l'enseignement, de l'éducation et de la formation), après deux années de tâtonnements, semblent plutôt renforcer et augmenter les tensions entre l'évaluation certificative institutionnelle, l'évaluation académique sommative du master universitaire et la velléité de maintenir une approche réflexive et formative de l'Espe. Les étudiants doivent, d'une part, se conformer aux examens universitaires pour obtenir le master et, d'autre part, accepter les contraintes imposées par l'inspection et les tuteurs pour se faire habiliter. La partie formative de l'Espe n'est quasiment pas prise en compte pour la titularisation avec pour conséquence le renforcement de l'évaluation certificative institutionnelle. Pourtant, la nouvelle réforme qui devrait ouvrir l'école au projet de type collaboratif, à l'interdisciplinarité et au numérique aurait bien besoin d'une modification profonde de la formation des nouveaux enseignants dans une société numérique en mouvement.

\section{BIBLIOGRAPHIE}

Allal, L. (2009). Pratiques évaluatives des enseignants face aux méthodologies des recherches évaluatives portant sur les systèmes scolaires. In L. Mottier Lopez \& M. Crahay (Ed.), Évaluations en tension : entre la régulation des apprentissages et le pilotage des systèmes (pp. 29-45). Bruxelles: De Boeck Supérieur.

Allal, L. (2013). Teachers' professional judgement in assessment: a cognitive act and a socially situated practice. Assessment in Education: Principles, Policy \& Practice (special issue: Moderation practice and teacher judgement), 20, 20-34.

Avodo, J. (2012). La qualification péjorative comme forme de violence verbale et d'agir professoral. Signes, Discours et Sociétés. En ligne : http://www.revue-signes.info/document.php? $\mathrm{id}=2539$.

Benveniste, E. (1974). Problèmes de linguistique générale, 2. Paris : Gallimard.

Boutet, J. (1994). Construire le sens. Paris : Peter Lang. 
Bres, J. (1998). Entendre des voix : de quelques marqueurs dialogiques en français. In Bres, J., Delamotte, R., Madray, M. \& Siblot, P. L'autre en discours, pp. 191-199.

Bres, J., \& Mellet, S. (2009). Une approche dialogique des faits grammaticaux, Langue française, 3, 163, 3-20.

Bucciarelli, L. (1994). Designing Engineers. Massachusetts Institute of Technology: Press paperback edition.

Bucheton, D. \& Chabanne, J.-C. (2002). Parler et écrire pour penser, apprendre et se construire. Paris : PUF.

Carcassonne, M. (2010). Quelles sont les significations dessinées par le dialogisme ? Une perspective en analyse de discours. Colloque international Dialogisme : langue, discours, Montpellier. En ligne : http://recherche.univ-montp3.fr/praxiling/spip.php?article264

Charaudeau, P. (1992). Grammaire du sens et de l'expression. Paris : Hachette.

Charaudeau, P. \& Maingueneau, D. (2002). Dictionnaire d'analyse du discours. Paris : Éditions du Seuil.

Cicurel, F. (1994). Marques et traces de la position de l'autre dans les discours d'enseignement des langues. In Les carnets du Cediscor, pp. 93-104. En ligne : http://cediscor.revues.org/582

Daille, B., Monceaux, L. \& Vernier, M. (2009). DEFT'09 : détection de la subjectivité et catégorisation de textes subjectifs par une approche mixte symbolique et statistique, 103-114. En ligne : https://deft.limsi.fr/actes/2009/pdf/8_vernier.pdf

Deum, M. \& Vanhulle, S. (2006). L'écriture réflexive en formation initiale des enseignants : entre réconciliation avec l'écrit et apprentissage de la rigueur conceptuelle. Langage \& pratiques, 37, 6-19.

Dillenbourg, P., Poirier, C. \& Carles, L. (2003). Communautés virtuelles d'apprentissage : e-jargon ou nouveau paradigme? In Taurisson, A. \& Sentini, A. Pédagogies. Net. Montréal Presses.

Draelants, H. (2007). Entre le pair et l'expert, trouver la distance qui convient. Une question de légitimation pour le conseiller pédagogique? Recherches sociologiques et anthropologiques, 38(1), 163-182.

Duru-Bellat, M. (2009). Evaluation externe du système éducatif et ressenti des acteurs. In L. Mottier Lopez \& M. Crahay (Ed.), Evaluations en tension. Entre la régulation des apprentissages et le pilotage des systèmes (pp. 47-59). Bruxelles : De Boeck Supérieur.

Figari, G. (1994). Evaluer : quel référentiel ? Bruxelles : De Boeck Université.

Figari, G. (2006). L'activité évaluative entre cognition et réponse sociale : nouveaux défis pour les évaluateurs. Mesure et Evaluation en Education, 29(1), 5-18.

Gilbert, M. (2008). La responsabilité collective et ses implications. Revue française de science politique, 6, vol. 58, 899-913.

Goffman, E. (1973). La mise en scène de la vie quotidienne, tome II. Paris : Minuit.

Gomez, F. (2001). Le mémoire professionnel. Objet de recherche et outil de formation. Bruxelles : De Boeck Université.

Grize J.-B. (1990). Logique et langage. Gap : Ophrys.

Huver, E. \& Springer, C. (2011). L'évaluation en langues. Paris : Didier. 
Lehti, L. (2010). Le positionnement intersubjectif dans les blogs des politiciens français. Etude de cas des interrogations. Colloque international Dialogisme : langue, discours, Montpellier. En ligne : http://recherche.univ-montp3.fr/praxiling/spip.php?article264

Longuet, F. (2012). L'impact des outils d'évaluation qualitative et du web 2.0 sur le développement et l'identification des compétences professionnelles des enseignants de langues. En ligne : http:// tel.archives-ouvertes.fr/tel-00770640/

Longuet, F. \& Springer, C. (2012). Développer et évaluer les compétences professionnelles des enseignants de langues à l'université : une mission impossible ? In Causa, M., ed. Formation initiale et profil d'enseignant de langues : enjeux et questionnements. (pp. 247-278). Bruxelles : De Boeck Supérieur.

Morrissette, J., Mottier Lopez, L., \& Tessaro, W. (2012). La production de savoirs négociés dans deux recherches collaboratives sur l'évaluation formative. In Mottier Lopez, L. \& Figari, G. (Ed.), Modélisations de l'évaluation en éducation. Questionnements épistémologiques (pp. 27-43). Bruxelles : De Boeck. En ligne : http://archive-ouverte.unige.ch/unige:23183

Morrissette, J. \& Compaoré, G. (2012). Le savoir-faire enseignant sur l'évaluation formative informelle. Formation et profession, 20 (3), 26-35.

Mottier Lopez, L. \& Crahay, M. (2009). Evaluations en tension : entre la régulation des apprentissages et le pilotage des systèmes. Bruxelles : De Boeck Supérieur.

Mottier Lopez, L., Tessaro, W., Dechamboux, L. \& Morales Villabona, F. (2012). La modération sociale : un dispositif soutenant l'émergence de savoirs négociés sur l'évaluation certificative des apprentissages des élèves. Questions Vives [En ligne], 6 (18), 159-175. En ligne : http:// questionsvives.revues.org/1235

Paquay, L. (Éd.). (2004). L'évaluation des enseignants : tensions et enjeux. Paris : L'Harmattan.

Perreau, L. (2010). En quel sens peut-on parler d'intentionnalité collective ? Bulletin d'analyse phénoménologique. VI 8, 213-229.

Peytard, J. (1990). Évaluation sociale dans les thèses de Mikhaïl Bakhtine et représentations de la langue. Langue française, 85, 6-21.

Quillard, V. (2001). La diversité des formes interrogatives : comment l'interpréter ? Langage et société, $1,95,57-72$.

Rabatel, A. (2004). L'effacement énonciatif dans les discours rapportés et ses effets pragmatiques. Langages, 156, 3-17.

Rabatel, A. (2008). Retour sur les relations entre locuteurs et énonciateurs Des voix et des points de vue. Recherches linguistiques, 32, 357-373. En ligne : https://halshs.archives-ouvertes.fr/ halshs-00504918/document

Rouveyrol, L. (1999). Pour une stylistique du « taxème » dans le débat politique télévisé : Analyse de quelques réseaux interactionnels signifiants. Asp., 99-119.

Schön, D. A. (1993). Le praticien réflexif. À la recherche du savoir caché dans l'agir professionnel. Montréal: Éditions logiques.

Schön, D. A. (1992). Design as reflective conversation with the materials of a design situation. Knowledge-Based Systems. 5 (1), 3-14.

Tessaro, W. (2015). Les épreuves externes comme soutien au développement professionnel des enseignants dans leurs pratiques d'évaluation certificative : les apports d'une recherche collaborative. Evaluer. Journal international de Recherche en Education et Formation, 1(2), 49-65. 
Therkelsen, R. (2009). Comment identifier une question polyphonique. Langue française, 4, 164, 113-122.

Vion, R. (2001). Effacement énonciatif et stratégies discursives. In De Mattia, M. \& Joly, A. De la syntaxe à la narratologie énonciative, pp. 331-354. Nice : Ophrys.

Voloshinov, V. (2010). Marxisme et philosophie du langage. Les problèmes fondamentaux de la méthode sociologique dans la science du langage. Paris : Lambert-Lucas.

Wenger, E. (2005). La théorie des communautés de pratique. Apprentissage, sens et identité. Québec : Les Presses de l'université Laval.

INDEX

Mots-clés : évaluation formative, évaluation certificative, didactique des langues, formation initiale, web 2.0, communauté de pratique

Keywords : formative evaluation, certificative evaluation, language didactics, initial training, community of practice, web 2.0

\section{AUTEUR}

\section{FRÉDÉRIQUE LONGUET}

Docteure, formatrice, ESPE Paris Sorbonne 